\title{
Erratum to: Mindlin's Micro-structural and Gradient Elasticity Theories and Their Thermodynamics
}

\author{
C. Broese ${ }^{1} \cdot$ C. Tsakmakis ${ }^{1} \cdot$ D. Beskos ${ }^{2}$
}

Published online: 27 May 2016

(C) Springer Science+Business Media Dordrecht 2016

\section{Erratum to: J Elast (2016)}

\section{DOI 10.1007/s10659-016-9572-7}

The signs in front of the terms $\partial_{j} \tilde{\phi}_{j k}$ and $h_{m n j k} \partial_{j} \phi_{m n}$ in Eq. (131), and in front of the surface integral in Eq. (137) should be "-", respectively. The correct forms of these equations are

$$
\begin{aligned}
F_{k} & =f_{k}-\partial_{j} \tilde{\phi}_{j k}=f_{k}-h_{m n j k} \partial_{j} \phi_{m n}, \\
W_{\text {inert. }} & =\int_{V} I_{k} \dot{u}_{k} d V=-\dot{\mathcal{T}}-\int_{\partial V} n_{j}\left(\tilde{i}_{j k} \dot{u}_{k}\right) d S .
\end{aligned}
$$

Consequential errors concern (138), (139), (170)-(173), (223), (246), (249). The correct forms of these equations read

$$
\begin{aligned}
& W_{\text {conv.body }}:=\int_{V} F_{k} \dot{u}_{k} d V=\int_{V}\left(f_{k} \dot{u}_{k}+\tilde{\phi}_{j k} \partial_{j} \dot{u}_{k}\right) d V-\int_{\partial V} n_{j}\left(\tilde{\phi}_{j k} \dot{u}_{k}\right) d S, \\
& \int_{V} b_{k} \dot{u}_{k} d V=W_{\text {inert. }}+W_{\text {conv.body }}=-\dot{\mathcal{T}}+W_{\text {conv.body }}-\int_{\partial V} n_{j}\left(\tilde{i}_{j k} \dot{u}_{k}\right) d S, \\
& W_{\text {gen.ext. }}=\int_{\partial V}\left(\hat{P}_{k}^{*} \dot{u}_{k}+\hat{R}_{k} D \dot{u}_{k}\right) d S-\int_{\partial V} n_{j}\left(\tilde{i}_{j k} \dot{u}_{k}\right) d S+W_{\text {conv.body }}-\dot{\mathcal{T}}, \\
& W_{\text {diff. }}=\int_{\partial V}\left[\left(\hat{P}_{k}^{*}-n_{j} \tilde{i}_{j k}\right) \dot{u}_{k}+\hat{R}_{k} D \dot{u}_{k}\right] d S+W_{\text {conv.body },}
\end{aligned}
$$

The online version of the original article can be found under doi:10.1007/s10659-016-9572-7.

C. Tsakmakis

tsakmakis@mechanik.tu-darmstadt.de

1 Department of Continuum Mechanics, Faculty of Civil Engineering, TU Darmstadt, Franziska-Braun-Str. 7, 64287, Darmstadt, Germany

2 Department of Civil Engineering, University of Patras, 265 00, Patras, Greece 
$\hat{P}_{k}=\hat{P}_{k}^{*}-n_{j} \tilde{i}_{j k}$

either $\hat{P}_{k}=\hat{P}_{k}^{*}-n_{j} \tilde{i}_{j k}$ or $u_{k}$,

$\hat{P}_{1} \equiv \hat{P}=n_{1} E\left(u_{, x}-g^{2} u_{, x x x}\right)+n_{1} \rho h^{2} u_{, x t t}$,

Version 1: $\quad\left[\bar{U}_{, \bar{x}}-\bar{g}^{2} \bar{U}_{, \bar{x} \bar{x} \bar{x}}-\bar{h}^{2} \bar{\omega}^{2} \bar{U}_{, \bar{x}}\right]_{\bar{x}=1}=\bar{\tau}_{0}$,

$\zeta=\left\{\begin{array}{ccc}1-\bar{h}^{2} \bar{\omega}^{2} & : & \text { Version } 1 \\ 1 & : & \text { Version } 2 .\end{array}\right.$

There are also consequential errors in the plots according to Model V1 in Figs. 2-5, 10, 11. The corrected plots are displayed in the remainder of this erratum. All comments pertaining to these figures remain valid, except of the following statement in Sect. 6.4, "It can be seen in Fig. 2, that ... to the $\bar{x}$-axis, than the corresponding V2 responses". The correct statement reads as follows: "It can be seen in Fig. 2, that for $\bar{\omega}=1.4$ the $\bar{U}$ values according to V1 and $\mathrm{V} 2$ are larger than those due to the classical solution and that the responses due to V1 are larger than those due to V2. But this is no more true for $\bar{\omega}=2 ; 3 ; 4$. The general tendency which can be stated in all Figs. $2-5$ is that, the V2-responses are always closer to the $\bar{x}$-axis, than the corresponding V1-responses." That is, in the corrected version, Models V1 and V2 switch their roles.

Finally, the last sentence in the conclusions should be corrected as follows: "it can be recognized, that for increasing values of $\bar{g}$ the responses $\bar{U}$ decrease in all cases and in particular the responses due to V3 are larger than those due to the other two models, meaning V3 is less stiff compared to the other two models".

It is emphasized, however, that all corrections made are formal and by no means affects the theoretical conclusions of the paper.

Fig. 2 Model responses for $\bar{\omega}=1.4, \bar{g}=0.0, \bar{\tau}_{0}=0.015$. Gradient effects arise only from inertia

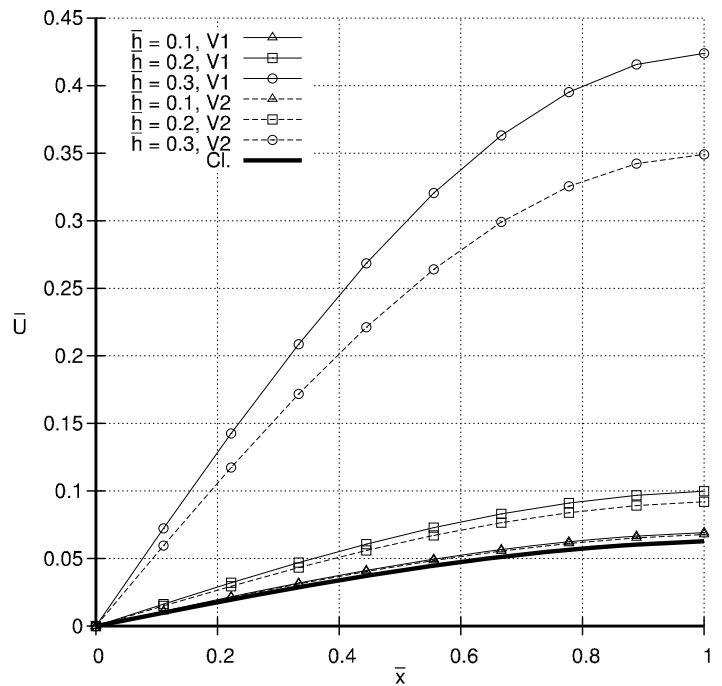


Fig. 3 Model responses for $\bar{\omega}=2.0, \bar{g}=0.0, \bar{\tau}_{0}=0.015$. Gradient effects arise only from inertia

Fig. 4 Model responses for $\bar{\omega}=3.0, \bar{g}=0.0, \bar{\tau}_{0}=0.015$. Gradient effects arise only from inertia

Fig. 5 Model responses for $\bar{\omega}=4.0, \bar{g}=0.0, \bar{\tau}_{0}=0.015$. Gradient effects arise only from inertia
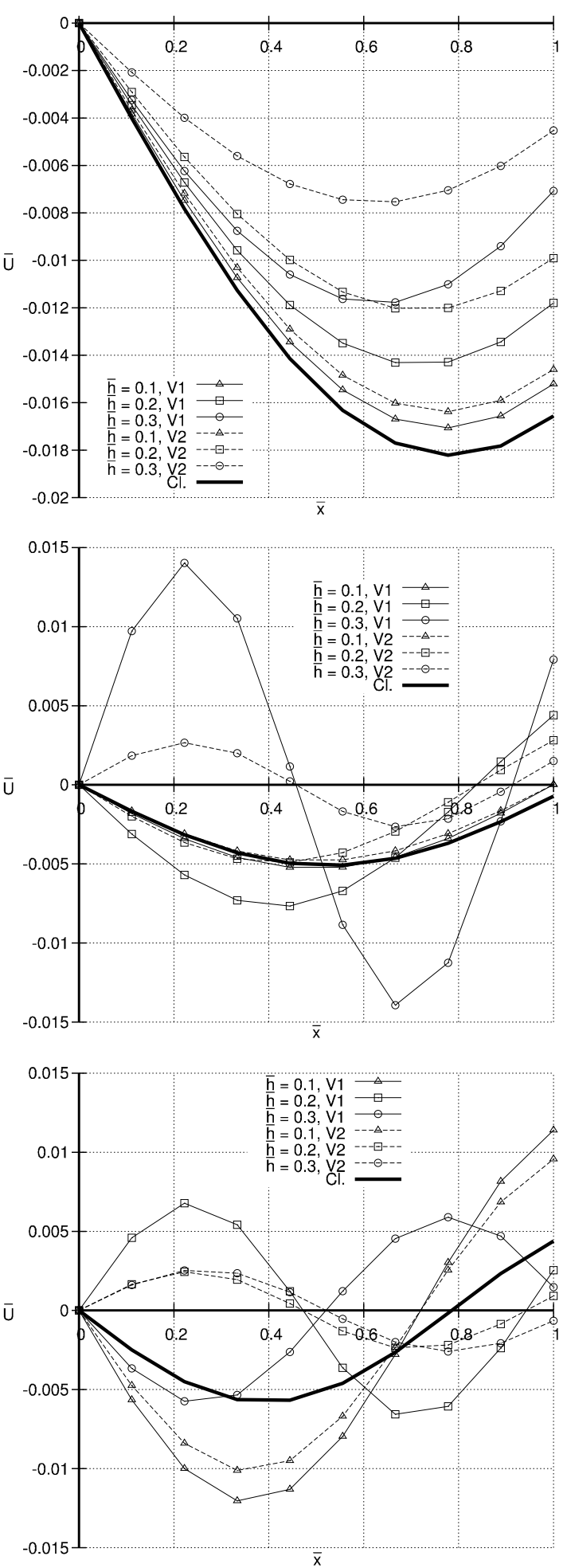
Fig. $10 \bar{\omega}=1.4, \bar{g}=0.2$, $\bar{\tau}_{0}=0.015$. Controversial effects of $\bar{g}$ and $\bar{h}$ on the responses

Fig. $11 \bar{\omega}=2.0, \bar{g}=0.2$, $\bar{\tau}_{0}=0.015$. Controversial effects of $\bar{g}$ and $\bar{h}$ on the responses
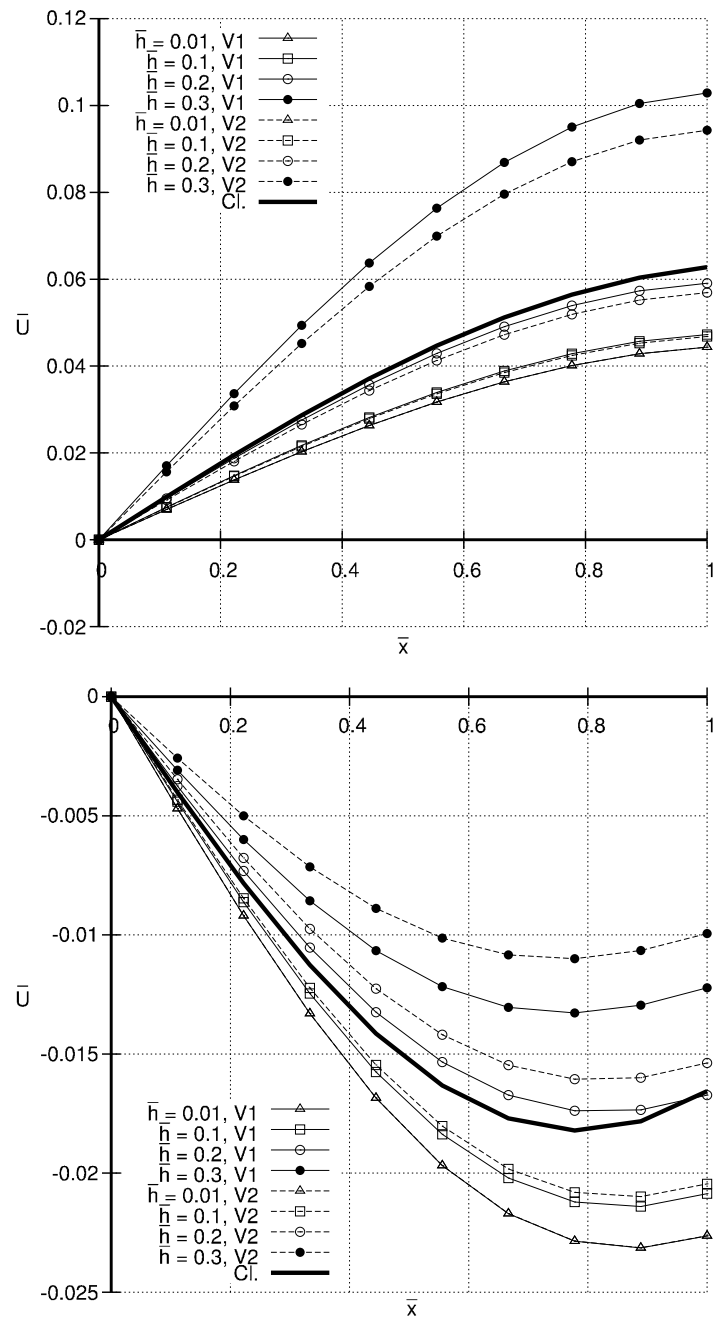